\title{
The environment exerts a greater influence than the transgene on the transcriptome of field-grown wheat expressing the $P m 3 b$ allele
}

\author{
Carolina Diaz Quijano • Susanne Brunner • \\ Beat Keller • Wilhelm Gruissem • \\ Christof Sautter
}

Received: 14 July 2013/Accepted: 15 July 2014/Published online: 6 August 2014

(C) Springer International Publishing Switzerland 2014

\begin{abstract}
Wheat provides $20 \%$ of the calories consumed worldwide. Powdery mildew infections of wheat can result in more than $30 \%$ yield loss but it has been demonstrated that wheat overexpressing Pm3b, an allele of the R gene $P m 3$, has enhanced resistance against powdery mildew under field conditions. A gene expression profile study using GeneChip Wheat Genome Array and Fluidigm 96.96 Dynamic Arrays was performed to obtain insights into the mode of action of $P m 3 b$ and to elucidate the molecular basis of pleiotropic effects observed in three out of four independent transgenic events under field conditions. A cluster analysis of the microarray data and a principal component analysis of the Fluidigm 96.96 Dynamic Arrays data showed that transgenic lines and null segregants grouped together. The microarray analysis of samples from fungicide-treated plants revealed that significantly fewer genes were differentially expressed in Pm3b\#1 than in Pm3b\#2, which had
\end{abstract}

Electronic supplementary material The online version of this article (doi:10.1007/s11248-014-9821-0) contains supplementary material, which is available to authorized users.

C. D. Quijano $(\bowtie) \cdot$ W. Gruissem · C. Sautter Plant Biotechnology, Department of Biology, ETH Zurich, Universitätstrasse 2, 8092 Zurich, Switzerland e-mail: carolina.diaz.quijano@alumni.ethz.ch

S. Brunner · B. Keller

Institute of Plant Biology, University of Zurich,

Zollikerstrasse 107, 8008 Zurich, Switzerland a pleiotropic phenotype in the field, compared to their null segregants. Together, our data provide evidence that the environment influenced gene expression in the Pm3b lines more than the transgene itself.

Keywords Triticum aestivum $\cdot$ Pm3b Pleiotropic effects $\cdot$ Expression profile $\cdot$ Microfluidic dynamic arrays

\section{Introduction}

Crop breeding programs have made extensive use of resistance genes ( $R$ genes) in the past decades. $R$ genes provide specific resistance to pathogens by recognizing pathogen effectors or their action on a host-cell target (Jones and Dangl 2006). The products of $R$ genes, the $\mathrm{R}$ proteins, can be classified into two main groups: the nucleotide binding (NB)-leucine-rich repeats (LRR) and extracellular LRR (e-LRR) (Chisholm et al. 2006). After recognizing pathogen effectors, $\mathrm{R}$ proteins give rise to a rapid and strong defense response, the effector-triggered immunity (ETI), which often involves triggering plant cell death through a hypersensitive response (Dodds and Rathjen 2010). Several reports have documented increased disease resistance in plants overexpressing $\mathrm{R}$ genes, including overexpression of Prf and Pto in tomato (Oldroyd and Staskawicz 1998; Tang et al. 1999), Xa3 in rice (Cao et al. 2007), and Bs2 from pepper in tomato (Tai et al. 1999). 
The powdery mildew resistance gene $P m 3$ encodes a protein of the subgroup of NB-LRR proteins, the coiled-coiled (CC) NB-LRR proteins. The gene was first mapped by Briggle and Sears (1966) and controls race-specific resistance to the fungal pathogen powdery mildew. Powdery mildew in wheat is caused by the ascomycete Blumeria graminis f. sp, a true obligate biotroph and is a continuous threat to wheat yield, producing losses of 13-34\% (Liu et al. 2001).

Pm3b, one of the seven alleles (Pm3a-g) of Pm3 identified using genetic approaches, was cloned (Yahiaoui et al. 2004) and later transformed into Bobwhite SH 98 26, a Mexican spring wheat breeding line (Brunner et al. 2011). We carried out leaf segment assays and three seasons of field trials to test the resistance of genetically modified (GM) plants expressing $P m 3 b$ under the control of the maize ubiquitin promoter (Brunner et al. 2011). In both leaf segment assays and field trials, four independent transformation events (Pm3b\#1, Pm3b\#2, Pm3b\#3, and $\mathrm{Pm} 3 \mathrm{~b} \# 4$ ) produced significantly less powdery mildew symptoms in GM plants than in true null segregant lines (Sb\#1, Sb\#2, Sb\#3 and Sb\#4) or the wild type. Apart from powdery mildew resistance, Pm3b\#1 was phenotypically similar to $\mathrm{Sb \# 1}$ in the field. In contrast, Pm3b\#2, Pm3b\#3 and Pm3b\#4 showed pleiotropic effects in the field trials that were not observed in the greenhouse or growth chambers: early yellowing of the leaves, reduced stomatal conductance, reduced plant height, and opened florets. Each of the effects was shared by at least two of the transformation events and all of the effects were displayed by Pm3b\#2. The penetrance of the pleiotropic effects, however, varied in different environments within the field (Brunner et al. 2011). Thus, it is conceivable that the transgene itself and not the transgene insertion site was responsible for the phenotypic changes, since they were not observed in the corresponding null segregant lines. Moreover, the transgenic line with the highest $P m 3 b$ expression showed the strongest pleiotropic effects. As similar effects were observed in independent transformation events, we hypothesize that the observed phenotypes are not related to the insertion site.

Here we present two independent gene expression profiles of the Pm3b\#1 and Pm3b\#2 transgenic lines compared to their null segregants $\mathrm{Sb \# 1}$ and $\mathrm{Sb \# 2,}$ obtained using Affymetrix Microarray and Fluidigm 96.96 Dynamic Arrays in order to investigate the molecular basis of the observed pleiotropic effects and to obtain insights into the plant pathogen response. Pm3b\#1 was selected because it showed the highest resistance to powdery mildew in the 2009 field trial but no pleiotropic phenotype (Brunner et al. 2011). In turn, Pm3b\#2 was selected because all plants showed pleiotropic effects and the highest $P m 3 b$ transcript abundance. Selected differentially expressed genes were further analyzed using Fluidigm 96.96 Dynamic Arrays IFCs (Integrated Fluidic Circuits) as a novel method to quantify transcript abundance in plants. Our results reveal that the environment exerts a greater influence than the transgene on the transcript profile.

\section{Materials and methods}

\section{Field experiments}

The field trials were performed by the wheat consortium within the frame of the National Research Program 59 (http://www.nrp59.ch). Field trials were performed at Agroscope Reckenholz-Tänikon (ART) (Zurich, Switzerland). The field tests as well as the artificial inoculation with powdery mildew have already been described in detail (Brunner et al. 2011).

\section{Plant material, RNA and cDNA}

Plant material was used from two lines corresponding to two different transformation events (Pm3b\#1 and Pm3b\#2) and their respective null segregant lines (Sb\#1 and Sb\#2). In the field seasons 2009 and 2010, three replicates of 15 leaves (leaf 5) were collected from both artificially inoculated and fungicide-treated micro-plots $(1.0 \times 1.3 \mathrm{~m})$ at the BBCH61-69 stage (flowering). Samples were frozen in liquid nitrogen immediately after collection in the field and stored at $-80{ }^{\circ} \mathrm{C}$ until RNA extraction.

RNA was extracted using TRIzol reagent (Invitrogen) according to the manufacturer's instructions. To avoid DNA contamination, RNA samples were treated with the RQ1 RNase-free DNase kit (Promega). The quality of the isolated RNA was determined with a NanoDrop ND 1000 (NanoDrop Technologies) and gel electrophoresis. Total RNA samples $(1.5 \mu \mathrm{g})$ were reverse-transcribed into double-stranded cDNA with a RevertAid First Strand cDNA Synthesis Kit (Fermentas). 
Microarray experiment

A total of 24 GeneChip Wheat Genome Arrays (Affymetrix Inc.) were hybridized using leaf samples from Pm3b\#1 and Pm3b\#2 and their null segregants, both artificially infected and fungicide-treated. Three biological replicates were collected in the field trial at Reckenholz in 2009. During analysis of the microarray results, we found a high signal for posphinothricin acetyltransferase (bar reporter gene) in the second

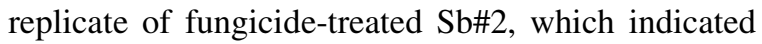
possible mixing of sample leaves with leaves from nearby GM plants expressing this selectable marker. Therefore, this replicate sample was not used for further analyses. The bar gene has been isolated from different Streptomyces species (Thompson et al. 1987; Wohlleben et al. 1988) and should not be a feature of the Affymetrix Wheat Genome Array, whose sequence information includes 2004 public content from Triticum aestivum UniGene Build \#38, ESTs from T. monococcum, T. turgidum, and Aegilops tauschii, and GenBank full-length mRNAs from all species. However, the Probe Set ID on NetAffx Analysis Center from Affymetrix (http://www. affymetrix.com/analysis/index.affx) is RPTR-TaX17220-1, has $100 \%$ homology to the sequence of the bar reporter gene, and is annotated as "Streptomyces hygroscopicus bar gene for posphinothricin acetyltransferase".

Hybridization and scanning of the microarrays were conducted at the Functional Genomics Center Zurich (FGCZ). Biotin-labelled cDNA samples $(15 \mu \mathrm{g})$ were fragmented randomly to $35-200 \mathrm{bp}$ at $94{ }^{\circ} \mathrm{C}$ in fragmentation buffer (Affymetrix Inc., P/N 900371) and added in $300 \mu \mathrm{l}$ of Hybridization Mix (Affymetrix Inc., P/N 900720) containing Hybridization Controls and Control Oligonucleotides B2 (Affymetrix Inc., P/N 900454). Samples were hybridized to GeneChip Wheat Genome Arrays for $16 \mathrm{~h}$ at $45^{\circ} \mathrm{C}$. Microarrays were then washed using an Affymetrix Fluidics Station 450 FS450_0001 protocol. An Affymetrix GeneChip Scanner 3000 (Affymetrix Inc.) was used to measure the fluorescence intensity emitted by the labeled target.

\section{Microarray analysis}

Raw data were processed using the Affymetrix AGCC software. Processed raw data obtained after scanning were normalized using the RMA algorithm of the B-Fabric analysis software (http://www.bfabric.org). Quality control measures were considered before performing the statistical analysis. For differential expression computation, B-Fabric applies the quantile normalization implemented in the Bioconductor package preprocessCore to make samples comparable (http://www.bioconductor.org/packages/devel/bioc/ $\mathrm{html} /$ preprocessCore.html). Probe sets with a false discovery rate (fdr)-adjusted $P$ value equal to or lower than 0.05 and a differential transcript abundance higher than twofold were used for further analysis. The FASTA sequence for each probe set was obtained through the NetAffx Analysis Center from Affymetrix (http://www.affymetrix.com/analysis/index.affx), analyzed by BLAST, and mapped and annotated using Blast2GO software (Conesa and Götz 2008). The Venn diagrams to describe the microarray analysis steps were obtained using the online tool BioVenn (Hulsen et al. 2008).

Fluidigm 96.96 Dynamic ArrayIFCs (Integrated Fluidic Circuits) experiment

Primer pairs were designed using the Primer-BLAST online tool at the NCBI website http://www.ncbi.nlm. nih.gov/tools/primer-blast/index.cgi, aiming at an amplicon length of 90-110 bp. Supplementary Table 2 shows the list of primers used in the experiment. Ta542297, Ta.2291 and Ta2776 were used as candidate reference genes for RT-qPCR data normalization (Paolacci et al. 2009).

Dynamic arrays were performed for samples from the field trials in 2009 and 2010. Both GM wheat lines Pm3b\#1 and Pm3b\#2, their respective null segregant

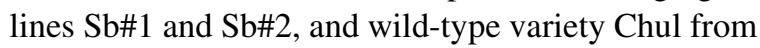
fungicide-treated and artificially inoculated plots were used. Two biological replicates from 2009 and three from 2010 field trials were used for each line. One artificially inoculated Pm3b\#2 sample from 2010 did not express $P m 3 b$, probably due to a sampling error at collection, and was removed from further analysis. The dynamic arrays were performed as described by Fluidigm 96.96 Dynamic Array IFCs (Integrated Fluidic Circuits) (PN 100-1208 B) with minor modifications: Total reaction volumes were changed: "STA (specific target amplification) reaction volume" was $6 \mu \mathrm{l}$ instead of $5 \mu \mathrm{l}$ and "sample pre-mix volume" was $8 \mu \mathrm{l}$ instead of $5 \mu \mathrm{l}$. The total volume per inlet 
chosen for the "assay mix" was $6 \mu \mathrm{l}$. The Biomark System was used to run the chips and data were collected with Fluidigm Real-Time PCR Analysis Software.

\section{Fluidigm 96.96 Dynamic Array IFCs data analysis}

Primers whose melting curves had more than one peak and therefore different melting temperatures $\left(T_{\mathrm{m}}\right)$ were eliminated from further analysis. $C t$ values were calculated by FluidigmReal-Time PCR Analysis Software. Linear derivative and automatic detector methods were set for baseline correction. PCR efficiencies were calculated with LinRegPCR (12.x) software and $\Delta C t$ calculated according to Karlen et al. (2007). The expression stability of the reference genes was checked using the geNorm algorithm within qbase ${ }^{\text {plus }}$ software, which recommends using only Ta.542297 and Ta.2291 as reference targets. Graphs and statistical analyses were performed using SigmaPlot for Windows Version 12.0 (2011, Systat Software, Inc.). Principal component analysis was performed using $\mathrm{R}$ language (http://www.R-project.org).

\section{Results}

Expression profiles from GM lines and respective null segregants are similar

As a first step, gene expression profiles of two different transformation events (Pm3b\#1 and Pm3b\#2) and their respective null segregant lines ( $\mathrm{Sb \# 1}$ and $\mathrm{Sb \# 2)}$ exposed to two different treatments were compared. Plants with and without fungicide application were infected with powdery mildew from spreader rows of infected plants (artificial inoculation). Changes in gene expression profiles obtained from microarray data were determined using cluster analysis (Fig. 1). Two clearly separated clusters were identified that corresponded to each of the treatments. Both transformation events were also distinguishable within these clusters. However, except for Pm3b\#2 and Sb\#2 that were artificially inoculated with powdery mildew, no other significant groupings were observed. This indicates that gene expression in the GM plants and their respective null segregant lines did not differ significantly.
Putative pleiotropic effects of transgene expression

We previously reported pleiotropic effects of the $P m 3 b$ transgene that resulted in similar pleiotropic phenotypes in three out of four transgenic Pm3 lines tested under field conditions (Brunner et al. 2011). In order to understand the molecular basis of the pleiotropic effects observed in line Pm3b\#2 (the line with the severest phenotype), a paired t-test of fungicide treated Pm3b\#1 and Pm3b\#2 replicas and their respective null segregant lines was performed. Only the results from fungicide-treated samples were used for this analysis in order to focus on the differences between GM and non-GM lines, independent of any effects of powdery mildew infection. For Pm3b\#1 compared to its null segregant line, none of the paired t-tested probe sets showed a false discovery rate (fdr)-adjusted $P$ value equal to or lower than 0.05 .

On the other hand, for Pm3b\#2 compared to its null segregant line, 379 probe sets showed a fdr-adjusted $P$ value equal to or lower than 0.05 and more than twofold differential expression. The annotations for the probes were obtained from NetAffix and those not annotated were blasted against the NCBI non-redundant protein database and mapped using Blast2GO (Conesa and Götz 2008). Only 98 of the significant 379 EST probes were annotated (Supplementary Table 1); seven had a transcript abundance lower and 91 had a transcript abundance higher in Pm3b\#2 than in $\mathrm{Sb \# 2} \mathrm{(Fig.} \mathrm{2).} \mathrm{Among} \mathrm{the} \mathrm{down-regulated}$ genes, the gene ontology (GO) categories were response to stress, secondary metabolic process, biosynthetic process, and response to external stimulus (Supplementary Figure 1A). Among the upregulated genes, the three process GO categories with highest representation were biosynthetic process, response to stress, and cellular protein modification process (Supplementary Figure 1B).

Of the 98 genes assigned to the EST probes mentioned above, 22 genes were selected to test their transcript abundance by Fluidigm Dynamic Array integrated fluidic circuits (IFCs). These genes were selected either because they were related to pathogen defense or could potentially explain the pleiotropic effects observed for Pm3b\#2 in the field (Supplementary Table 2). In addition to both GM wheat lines and their respective null segregant lines, transcript abundance was also measured in the wild-type variety Chul. 
Fig. 1 Cluster analysis of the expression profiles of Pm3b\#1, Pm3b\#2, and null segregant lines from the 2009 field trial obtained by microarray analysis.

Samples separate clearly according to treatment and transformation event, but not to genotype

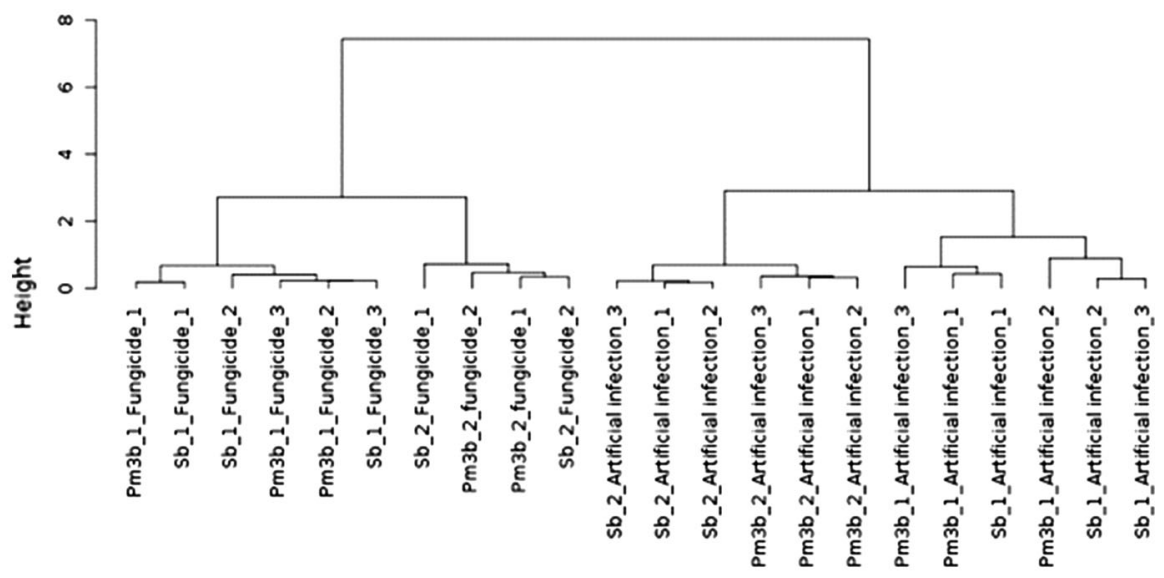

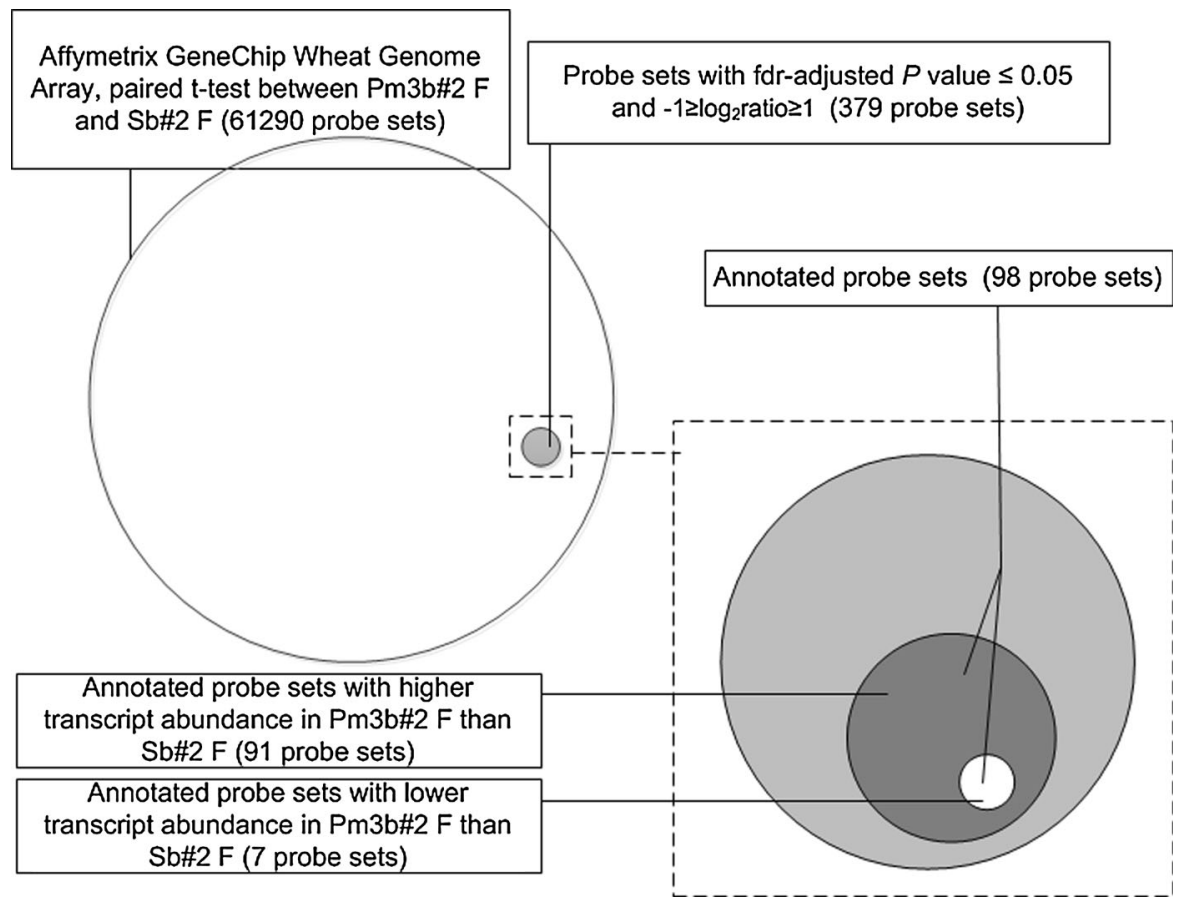

Fig. 2 Venn diagrams of microarray analysis to search for putative pleiotropic effects of transgene expression in Pm3b\#2. A paired t-test analysis was performed on Pm3b\#2 F versus its

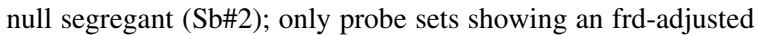
$P<0.05$ and $a-1 \geq \log 2$ ratio $\geq 1$ were selected for further

A comparison of the Affymetrix microarrays and Fluidigm arrays showed that $54.5 \%$ of the transcript measurements gave the same relative result, i.e. either higher or lower transcript abundance in Pm3b\#2 than in $\mathrm{Sb \# 2}$. However, comparison of the $\log _{2}$ (ratio) between Pm3b\#2 and Sb\#2 indicated a non-significant correlation of the two methods (tested by both analysis. Out of this list of probe sets only those with annotation on NetAffyx or on the NCBI non-redundant protein database were selected; 98 probe sets of which 91 showed higher and seven lower transcript abundance in Pm3b\# than in Sb\#2. $F$ fungicide-treated; $A I$ artificially inoculated

Pearson's and Spearman's correlation coefficients). The differences in the results could be due to the specificities of each method. Microarray data are error prone for multigene families because their transcripts could hybridize to similar probes in the array. In contrast, specific primers for qPCR are designed for each tested gene. Moreover, the specificity of primers 


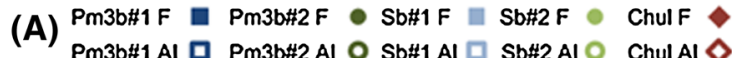

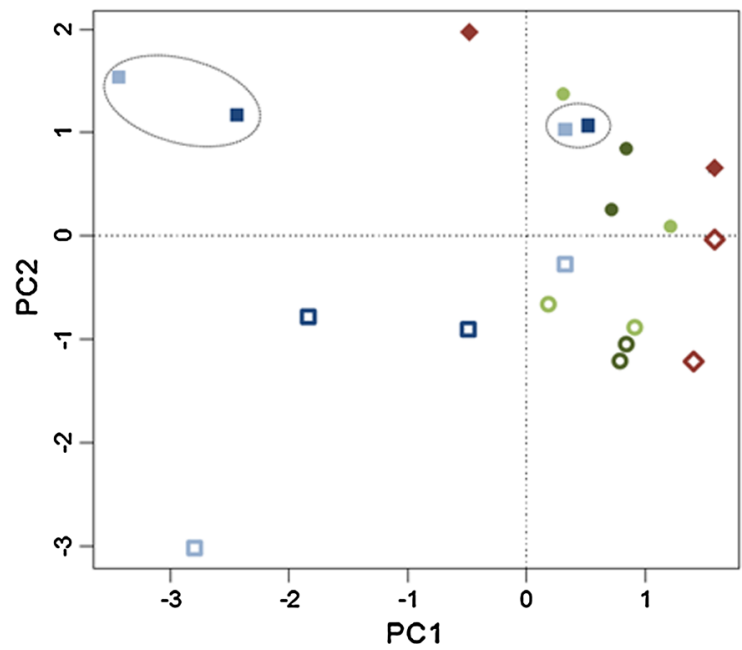

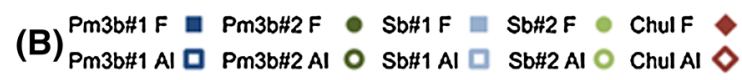

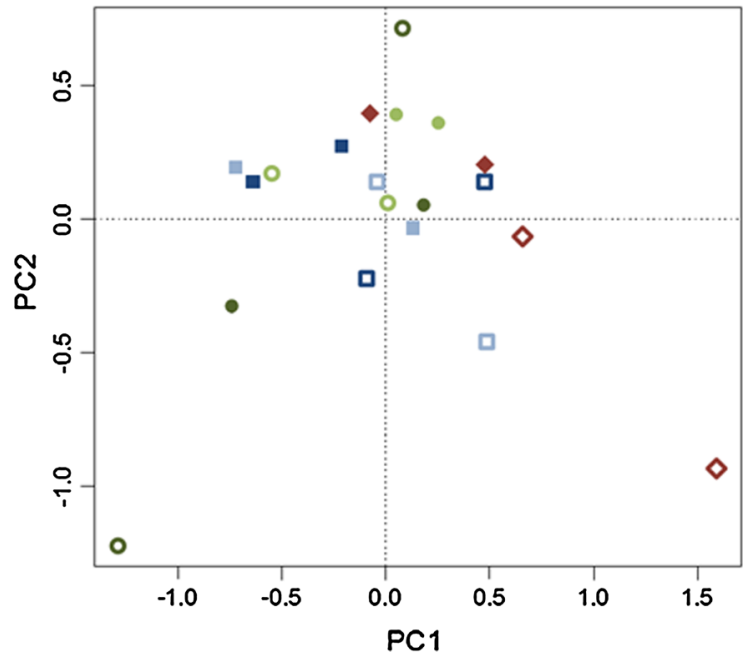

Fig. 3 Principal component analysis (PCA) of the mRNA abundance of genes tested in quantitative RT-PCR assays (a) 2009 field trial: the grouping of samples is similar to the microarray analysis (Fig. 1). (b) 2010 field trial: samples do not show a clear grouping pattern. Samples from the same replicate that group together are circled. $A I$ artificial inoculation with powdery mildew; $F$ fungicide-treated

is confirmed using melting curves, gel electrophoresis, and/or sequencing data of PCR products.

Despite the differences between the measurements from the two methods, a principal component analysis obtained using the Fluidigm Dynamic Array IFCs data of both fungicide-treated and artificially inoculated GM lines, their respective null segregants, and Chul showed grouping patterns similar to the cluster constructed with the Affymetrix microarray (Fig. 3a). The first principal component (PC1) accounted for $49.4 \%$ of the variation and showed a tendency to separate Pm3b\#1 and its null segregant line from Pm3b\#2 and its null segregant line. This tendency was clearer in the artificially inoculated than in the fungicide-treated samples. The putative homolog of the kinetochore protein SKP1 (S-phase kinase associated protein) made the largest contribution to the variation along PC1 and was negatively correlated with this component. The second principal component (PC2) accounted for $33.8 \%$ of the variation and distinguished artificially inoculated and fungicidetreated samples. The chalcone synthase gene made the largest contribution to variation along PC2 and was negatively correlated with this component. Interestingly, fungicide-treated Pm3b\#1 and Sb\#1 samples from the same replicate were more similar than were replicas from the same line, either Pm3b\#1 or Sb\#1. Therefore, for Pm3b\#1, block and treatment effects were more pronounced than the presence or absence of the transgene. This was not the case for Pm3b\#2.

The transcript abundance of these same 22 genes was measured in samples collected on the field in 2010 and a PCA was performed (Fig. 3b). The first principal component (PC1) accounted for $74.1 \%$ of the variation and skpl made the largest contribution to the variation along PC1 and was negatively correlated with this component. The second principal component (PC2) accounted for $13.3 \%$ of the variation and the chalcone synthase gene made the largest contribution to variation along PC2 and was negatively correlated with this component. Even when the genes contributing to the variation in the PCAs for both years were the same, no grouping patterns were observed in the PCA with the 2010 samples. This suggested that the expression profiles in both years differed for the different lines, indicating a stronger effect of the environment than the presence or absence of the transgene.

Differences between expression profiles of GM and non-GM lines under artificial inoculation conditions

Since the null segregant lines are truly isogenic to the transgenic Pm3 lines, they are the best material to investigate the effects of the race-specific resistance 
Fig. 4 Venn diagrams of microarray analysis to search for putative defense pathways activated by $P m 3 b$. A paired t-test analysis was performed on Pm3b\#1 AI versus Pm3b\#1 F (a) and an independent analysis on Pm3b\#2 AI versus Pm3b\#2 F (b). Only the probe sets showing an frd-adjusted $P<0.05$ and a $-1 \geq \log 2$ ratio $\geq 1$ were selected for further analysis. These two lists were compared to those obtained from paired t-tests analysis performed on $\mathrm{Sb \# 1} \mathrm{AI}$ versus $\mathrm{Sb \# 1} \mathrm{F}$ and $\mathrm{Sb \# 2} \mathrm{AI}$ versus $\mathrm{Sb \# 2} \mathrm{F,} \mathrm{respectively.}$ Those probe sets with the same regulation pattern were subtracted from the original Pm $3 b \# 1$ and Pm3b\#2 lists. This substraction was performed to ensure that the probe sets analyzed further would be those exclusively up- or downregulated in GM lines after artificial inoculation. Moreover, only those with annotation on NetAffyx or on the NCBI non-redundant protein database were selected. $F$ fungicidetreated, $A I$ artificially inoculated
(A)

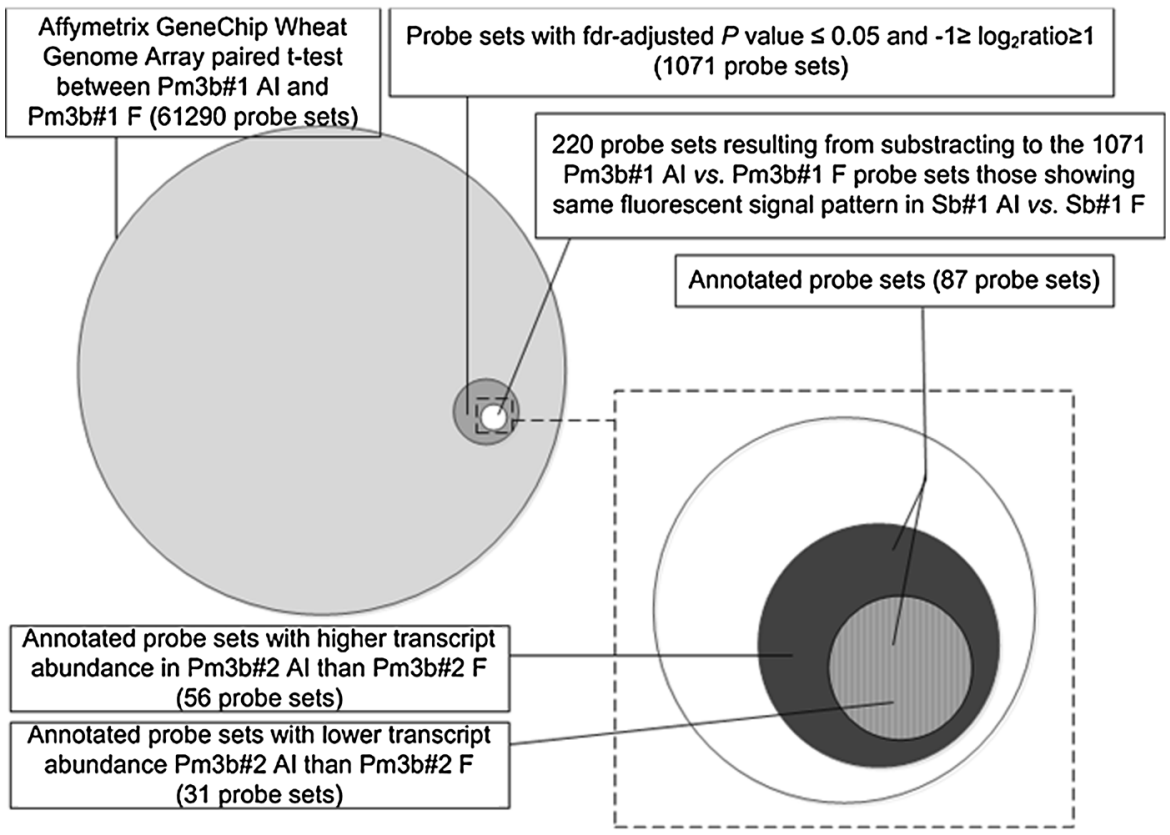

(B)

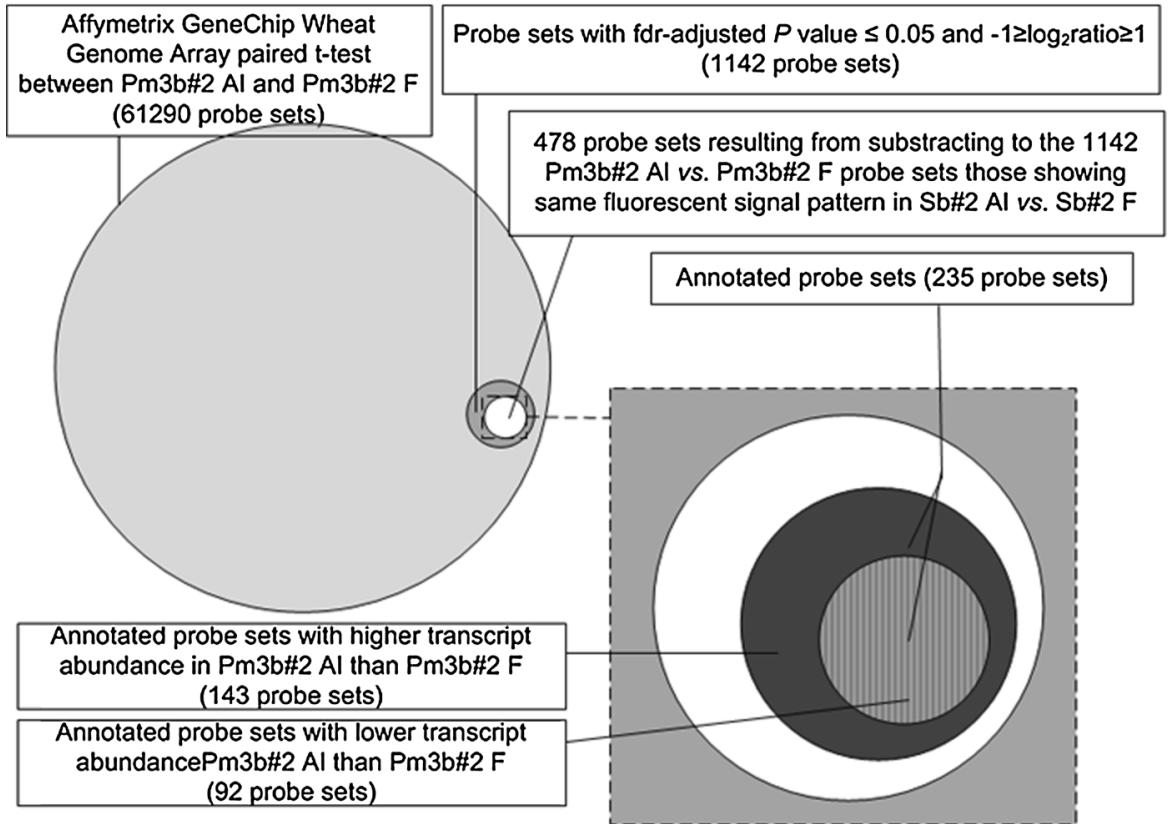

gene $P m 3 b$. To obtain insights into pathogen defense mechanisms mediated by $\mathrm{Pm} 3 \mathrm{~b}$, we analyzed the gene expression data from artificially inoculated Pm3b\#1 and Pm $3 \mathrm{~b} \# 2$ and their respective null segregant lines (Fig. 4). We performed four paired t-tests on the Affymetrix microarray data of artificially inoculated versus fungicide-treated plants: (1) artificially inoculated Pm3b\#1 versus fungicide-treated Pm3b\#1, (2) artificially inoculated Pm3b\#2 versus fungicide-treated Pm3b\#2, (3) artificially inoculated Sb\#1 versus

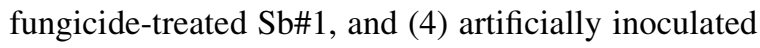
$\mathrm{Sb \# 2}$ versus fungicide-treated $\mathrm{Sb \# 2}$. In the microarray 
dataset, 1071 and 1142 probe sets showed signals in analyses (1) and (2), respectively that corresponded to an fdr-adjusted $P$ value equal to or lower than 0.05 and more than twofold differential expression. In order to focus exclusively on artificially inoculated Pm3b\#1 and Pm3b\#2, probe sets showing the same fluorescent signal pattern in analyses (1) and (3) or (2) and (4), i.e. present in both artificially inoculated GM lines and their respective artificially inoculated null segregants, were eliminated from further analysis. As a result, we obtained two lists of 220 and 478 probe sets with exclusively higher or lower transcript abundance in artificially inoculated Pm3b\#1 or Pm3b\#2, respectively. Among the 220 remaining probe sets from the Pm3b\#1 list, 87 were annotated (Supplementary Table 3). Of these, 56 genes had higher transcript abundance and 31 lower transcript abundance in artificially inoculated than fungicide-treated plants. The process GO categories with highest representation for genes with higher transcript abundance were defense response, protein phosphorylation, oxidation-reduction process, and auxin biosynthetic process (Supplementary Figure 2A). For the genes with lower transcript abundance, the categories were carbohydrate metabolic process, plastid organization, regulation of transcription, and DNA-dependent (Supplementary Figure 3A).

Among the remaining 478 probe sets from the Pm3b\#2 list, 235 genes were annotated (Supplementary Table 4); 143 had higher and 92 lower transcript abundance in artificially inoculated than fungicidetreated plants. The three most significant process GO categories for genes with higher transcript abundance were catabolic process, carbohydrate metabolic process, and response to stress (Supplementary Figure 2B). For the genes with lower transcript abundance, the three most significant GO categories were carbohydrate metabolic process, biosynthetic process, and transport (Supplementary Figures 3B). Genes corresponding to the category "response to stress" are over-represented among the down-regulated compared with the up-regulated genes.

In order to identify genes that might explain the increased resistance to powdery mildew infection in the $P m 3 b$ lines, we compared the two transformation event lists. Where the deregulation of a gene in an artificially inoculated compared with a fungicidetreated Pm 3 b sample is caused by $P m 3 b$ expression, it should be observed in both transformation events. If this is not the case, the cause of differential expression is more likely to lie with the site of insertion or the level of $P m 3 b$ transgene expression than with the transgene per se. Our data revealed eight genes with the same and two with opposite expression patterns in both transformation events (Table 1).

\section{Discussion}

Our data support previous reports that large differences in transcript abundance of non-target genes are not to be expected between GM and control lines (Gregersen et al. 2005; Baudo et al. 2006; Coll et al. 2010; Kogel et al. 2010). In the microarray analysis of the fungicide-treated wheat plants described here, no genes were found that had significantly higher or lower transcript abundance in Pm3b\#1 than in the corresponding null segregant control line and only a few genes in the case of Pm3b\#2, despite the pleiotropic phenotype displayed by the Pm3b\#2 line in the field (Brunner et al. 2011). As expected, a higher number of genes were differentially regulated when the GM and null segregant lines were inoculated with powdery mildew isolate 69224 , to which $P m 3 b$ confers race-specific resistance (Brunner et al. 2011). In fact, the cluster analysis of the microarray data showed clear grouping of the samples according to their artificial powdery mildew inoculation or fungicide application treatments, but not according to the presence or absence of the $P m 3 b$ transgene.

PCA of the qPCR data confirmed grouping of the analyzed samples according to treatment. The transgenic Pm3b lines and their null segregants did not cluster separately, indicating that their expression profiles are similar and independent of the presence of Pm3b. Moreover, the PCA patterns of the 2009 samples were different from those of the 2010 samples, although the same genotypes (or lines) were tested in both years. This suggests that the environment has a stronger effect on the transcriptome than the $P m 3 b$ transgene itself. Our results are thus consistent with a recent report by Barros et al. (2010), who found in maize that environmental factors caused more variation than the different genotypes, not only in their transcript profiles but also in their proteomes and metabolomes. Also, Baker et al. (2006) 
Table 1 Affymetrix microarray

\begin{tabular}{|c|c|c|c|c|}
\hline ProbeName & Seq. description & $\log 2$ ratio & $p$ value & $\mathrm{fdr}$ \\
\hline \multicolumn{5}{|l|}{ (A) } \\
\hline Ta.30950.1.A1_x_at & Glycosyl hydrolase & -1.593 & $2.68 \mathrm{E}-05$ & 0.001738 \\
\hline Ta.20785.1.S1_at & Pentatricopeptide repeat-containing protein & -1.225 & $2.18 \mathrm{E}-04$ & 0.006712 \\
\hline TaAffx.98097.1.A1_at & DNA polymerase i 3-5 exo domain & -1.006 & $2.24 \mathrm{E}-04$ & 0.00684 \\
\hline Ta.3784.2.S1_at & in $2-1$ protein & -1.63 & $4.51 \mathrm{E}-04$ & 0.01018 \\
\hline Ta.26051.1.A1_at & Pumilio-family rna binding repeat containing expressed & -1.021 & $8.48 \mathrm{E}-04$ & 0.01393 \\
\hline Ta.425.2.S1_at & 1-aminocyclopropane-1-carboxylate oxidase & 1.957 & $3.67 \mathrm{E}-04$ & 0.009067 \\
\hline Ta.3828.3.A1_a_at & Beta glucanase & 1.027 & $6.94 \mathrm{E}-03$ & 0.04009 \\
\hline $\begin{array}{l}\text { TaAffx.78001.1.S1_at } \\
\text { (B) }\end{array}$ & \multicolumn{3}{|c|}{ (B) } & 0.04219 \\
\hline Ta.9107.2.S1_a_at & 1-aminocyclopropane-1-carboxylate oxidase & -1.445 & $2.73 \mathrm{E}-04$ & 0.007523 \\
\hline Ta.4117.1.S1_x_at & Glycosyltransferase, HGA-like, putative, expressed & -1.1 & $3.69 \mathrm{E}-04$ & 0.009084 \\
\hline
\end{tabular}

Genes that showed equal (A) or opposite (B) expression patterns in both artificially inoculated Pm3b\#1 and artificially inoculated $\operatorname{Pm} 3 \mathrm{~b} \# 2$

observed greater differences in metabolic profile between different sites and years than between GM wheat and control lines.

S-phase kinase-associated protein 1 (SKP1) contributed most to the variation along PC1 of the PCA performed with 2009 samples and was, thus, responsible for the separation of Pm3b\#1 and its null segregant line from Pm3b\#2 and its null segregant line. This gene is a component of a Skp1-Cullin1-Fbox (SCF) complex that facilitates ubiquitin-mediated protein degradation. It was shown in Arabidopsis that SCF targeted an NLR R protein for degradation, preventing its over-accumulation and, thus, autoimmune responses (Cheng et al. 2011). In our study of samples from 2009, transcript abundance of $s k p l$ was higher in Pm3b\#2 than Pm3b\#1. This indicates that a plant mechanism controlling NLR R protein abundance and preventing autoimmune responses was less activated in Pm3b\#2 than in Pm3b\#1. This suggests that a combination of three factors was responsible for the Pm3b\#2 phenotype: (1) the higher $P m 3 b$ transcript abundance in Pm3b\#2, (2) lower down-regulation of the $\mathrm{R}$ protein, and (3) the fact that $\mathrm{Pm} 3 \mathrm{~b}$ is an $\mathrm{R}$ protein itself. On the other hand, the lack of grouping patterns in the PCA of the 2010 samples, despite the fact that Skpl also showed the largest variation along the PC, and thus accounted for the highest variation in the PCA, makes this enzyme a weak candidate to explain the observed pleiotropic effects. However, it is also possible that the plants adapted to the different environmental conditions independent of the transgene, which would be consistent with small changes in transcriptome profiles in the GM and null segregant lines. Thus, the effects of the $P m 3 b$ transgene on the transcriptome of the Pm3b lines could be a consequence of the transgene insertion and/or transcript abundance, as well as any interaction that these two factors may have with the environment.

In turn, the chalcone synthase gene contributed most to the variation along PC2 of the PCA performed with 2009 samples and, therefore, was responsible for the separation between artificially inoculated and fungicide-treated samples. Chalcone synthase is a key enzyme of the flavonoid/isoflavonoid biosynthesis pathway and is activated under stress conditions (Dao et al. 2011). Since there was no evident clustering separating Pm3b from null segregant lines in the artificially inoculated samples, this gene is most likely part of a general immune response and does not shed light on the defense pathways activated by $\mathrm{Pm} 3$.

Even though both transformation events provided increased resistance to the pathogen (Brunner et al. 2011), their expression profiles under artificial inoculation differed. Despite the higher number of genes differentially regulated exclusively in artificially inoculated $P m 3 b$ lines, compared to fungicide-treated lines, only eight genes had either significantly higher or lower transcript abundance in both transformation events in the Affymetrix microarray. Therefore, given that both transformation events share the same R gene, 
these results provide evidence that the influence of the environment on the expression profiles of artificially inoculated Pm3 lines is greater than that of the transgene.

In conclusion, our work provides evidence that the environment had a stronger influence on the transcriptome profiles of Pm3b\#1 and Pm3b\#2 lines in the field compared to their respective null segregants than the $P m 3 b$ transgene itself, not only in fungicide-treated plants but also in plants artificially inoculated with powdery mildew. The fact that we found only a few genes with consistent differential expression patterns in both Pm3b\#1 and Pm3b\#2 lines indicates that the effect of $P m 3 b$ on the transcriptome is probably not related to its function as an $\mathrm{R}$ gene per se.

Acknowledgments We thank Hubert Rehrauer, Jelena Kühn Georgijevic, and Catharine Aquino of the Functional Genomics Center Zurich (FGCZ) for microarray hybridization and support with data analysis. We are grateful to Aria Minder and Tania Torossi from the Genetic Diversity Centre (GDC) ETH Zurich for their technical advice on the Fluidigm Dynamic Arrays. We also thank our colleagues at the Swiss National Research Station, Agroscope Reckenholz-Tänikon ART for their support of the field experiment, particularly Carolin Luginbuehl and Andrea Foetzki for technical and scientific coordination. We gratefully acknowledge Patrick King for his help with the English. This project was supported by the Swiss National Science Foundation and was part of the Wheat Consortium, a collaborative research project of the Swiss National Research Programme 59 'Benefits and risks of the deliberate release of genetically modified plants' (http://www.NRP59.ch).

\section{References}

Baker JM, Hawkins ND, Ward JL, Lovegrove A, Napier JA, Shewry PR, Beale MH (2006) A metabolomic study of substantial equivalence of field-grown genetically modified wheat. Plant Biotechnol J 4:381-392

Barros E, Lezar S, Anttonen MJ, Van Dijk JP, Röhlig RM, Kok EJ, Engel K-H (2010) Comparison of two GM maize varieties with a near-isogenic non-GM variety using transcriptomics, proteomics and metabolomics. Plant Biotechnol J 8:436-451

Baudo MM, Lyons R, Powers S, Pastori GM, Edwards KJ, Holdsworth MJ, Shewry PR (2006) Transgenesis has less impact on the transcriptome of wheat grain than conventional breeding. Plant Biotechnol J 4:369-380

Briggle LW, Sears ER (1966) Linkage of resistance to Erysiphe graminis $\mathrm{f}$ sp. tritici $(\mathrm{Pm} 3)$ and Hairy Glume $(\mathrm{Hg})$ on Chromosome 1A of Wheat. Crop Sci 6:559-561

Brunner S, Hurni S, Herren G, Kalinina O, Von Burg S, Zeller SL, Schmid B, Winzeler M, Keller B (2011) Transgenic
Pm3b wheat lines show resistance to powdery mildew in the field. Plant Biotechnol J 9:897-910

Cao Y, Ding X, Cai M, Zhao J, Lin Y, Li X, Xu C, Wang S (2007) The expression pattern of a rice disease resistance gene $\mathrm{Xa} 3 / \mathrm{Xa} 26$ is differentially regulated by the genetic backgrounds and developmental stages that influence its function. Genetics 177:523-533

Cheng YT, Lib Y, Huanga S, Huanga Y, Dongd X, Zhangb Y, Lia X (2011) Stability of plant immune-receptor resistance proteins is controlled by SKP1-CULLIN1-F-box (SCF)mediated protein degradation. Proc Natl Acad Sci USA 108:14694-14699

Chisholm ST, Coaker G, Day B, Staskawicz BJ (2006) Hostmicrobe interactions: shaping the evolution of the plant immune response. Cell 124:803-814

Coll A, Nadal A, Collado R, Capellades G, Kubista M, Messeguer J, Pla M (2010) Natural variation explains most transcriptomic changes among maize plants of MON810 and comparable non-GM varieties subjected to two N-fertilization farming practices. Plant Mol Biol 73:349-362

Conesa A, Gotz S (2008) Blast2GO: a comprehensive suite for functional analysis in plant genomics. Int J Plant Genomics. doi:10.1155/2008/619832

Dao TTH, Linthorst HJM, Verpoorte R (2011) Chalcone synthase and its functions in plant resistance. Phytochem Rev 10:397-412

Dodds PN, Rathjen JP (2010) Plant immunity: towards an integrated view of plant-pathogen interactions. Nat Rev Genet 11:539-548

Gregersen PL, Brinch-Pedersen H, Holm PB (2005) A microarray-based comparative analysis of gene expression profiles during grain development in transgenic and wild type wheat. Transgenic Res 14:887-905

Hulsen T, de Vlieg J, Alkema W (2008) BioVenn-a web application for the comparison and visualization of biological lists using area-proportional Venn diagrams. BMC Genom 9:488

Jones JD, Dangl JL (2006) The plant immune system. Nature 444:323-329

Karlen Y, McNair A, Perseguers S, Mazza C, Mermod N (2007) Statistical significance of quantitative PCR. BMC Bioinform 8:131-146

Kogel KH, Voll LM, Schäfer P, Jansen C, Wu Y, Langen G, Imani J, Hofmann J, Schmiedl A, Sonnewald S, von Wettstein D, Cook RJ, Sonnewald U (2010) Transcriptome and metabolome profiling of field-grown transgenic barley lack induced differences but show cultivar-specific variances. Proc Natl Acad Sci USA 107:6198-6203

Liu S, Griffey CA, Maroof MAS (2001) Identification of molecular markers associated with adult plant resistance to powdery mildew in common wheat cultivar massey. Crop Sci 41:1268-1275

Oldroyd GED, Staskawicz BJ (1998) Genetically engineered broad-spectrum disease resistance in tomato. Proc Natl Acad Sci USA 95:10300-10305

Paolacci AR, Tanzarella OA, Porceddu E, Ciaffi M (2009) Identification and validation of reference genes for quantitative RT-PCR normalization in wheat. BMC Mol Biol 10:11

Tai TH, Dahlbeck D, Clark ET, Gajiwala P, Pasion R, Whalen MC, Stall RE, Staskawicz BJ (1999) Expression of the Bs2 
pepper gene confers resistance to bacterial spot disease in tomato. Proc Natl Acad Sci USA 96:14153-14158

Tang X, Xie M, Kim JY, Zhou J, Klessig DF, Martin GB (1999) Overexpression of Pto activates defense responses and confers broad resistance. Plant Cell 11:15-29

Thompson CJ, Rao Movva N, Tizard R, Crameri R, Davies JE, Lauwereys M, Botterman J (1987) Characterization of the herbicide-resistance gene bar from Streptomyces hygroscopicus. EMBO J 6(9):2519-2523
Wohlleben W, Arnold W, Broer I, Hillemann D, Strauch E, Punier A (1988) Nucleotide sequence of the phosphinothricin N-acetyltransferase gene from Streptomyces viridochromogenes Tü494 and its expression in Nicotiana tabacum. Gene 70:25-37

Yahiaou N, Srichumpa P, Dudler R, Keller B (2004) Genome analysis at different ploidy levels allows cloning of the powdery mildew resistance gene Pm3b from hexaploid wheat. Plant J 37:528-538 